\title{
PIVKA-II level is correlated to development of portal vein tumor thrombus in patients with HBV-related hepatocellular carcinoma
}

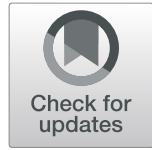

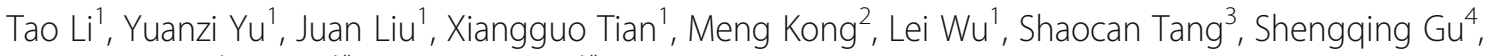
Jingfang Zhao ${ }^{1}, \mathrm{Yi} \mathrm{Cui}^{{ }^{*}}$ and Jinhua $\mathrm{Hu}^{1 *}$

\begin{abstract}
Aim: To evaluate the correlation of serum PIVKA-II levels and development of portal vein tumor thrombus (PVTT) in hepatocellular carcinoma (HCC) patients.

Methods: One hundred and twenty-three patients with newly diagnosed HCC were included in this study between March 2016 and October 2018. Thirty-five of these patients were detected with PVIT and all subjects were randomly divided to analysis group $(N=73)$ and validation $(N=50)$ group. Serum levels of PIVKA-II, laboratory tests including serum aspartate aminotransferase, total bilirubin, platelet count, albumin levels were demonstrated in all the patients. T-test, chi-squared test and logistic regression was used for analyzing data. Diagnostic efficiency and cut-off value of PIVKA-II in PVIT development of HCC patients were calculated using receiver operator curve (ROC) analysis.

Results: Serum level of PIVKA-II in HCC patients with PVTT was significantly higher than that in HCC patients without PVTT (995.8 $\mathrm{mAU} / \mathrm{ml}$ vs $94.87 \mathrm{mAU} / \mathrm{ml} ; P=0.003)$, as well as D-dimer levels $(2.12 \mathrm{mg} / \mathrm{L}$ vs $0.56 \mathrm{mg} / \mathrm{L} P=$ 0.001). Univariate analysis showed that high serum D-dimer level was an independent risk factor for development of PVIT (OR $=1.22,95 \% \mathrm{Cl} 1.02-1.45)$. ROC curve showed that among analysis group, the area under ROC curve (AUROC) of PIVKA-II was 0.73 (95\%CI 0.59-0.86). For the detection of PVTT in HCC, PIVKA-II had a sensitivity of 83.7\% and a specificity of $69.2 \%$ at a cutoff of $221.26 \mathrm{mAU} / \mathrm{ml}$, which had a sensitivity of $85.71 \%$ and a specificity of $55.56 \%$ in validation group, respectively.

Conclusion: Serum PIVKA-II level is a potential marker for diagnosis of PVTT in HCC patients, which may guide therapeutic strategy and assessment of tumor prognosis of HCC.
\end{abstract}

Keywords: Hepatocellular carcinoma, PIVKA-II, Portal vein tumor thrombus

\section{Introduction}

As the fifth most common cancer and the second highest cause of cancer-related death, hepatocellular carcinoma (HCC) is a severe health problem all over the world [1]. According to a recent research, more than one-third of HCC are diagnosed with portal vein tumor thrombosis (PVTT) or extrahepatic metastasis at BCLC C stage [2]. Prognosis of HCC patients with PVTT is especially poor, with a median survival of 3 months if no

\footnotetext{
*Correspondence: 15168886161@163.com; 386860155@qq.com

${ }^{1}$ Department of Gastroenterology, Provincial Hospital Affiliated to Shandong

University, 324, Jing 5 Rd, Jinan 250021, Shandong Province, China

Full list of author information is available at the end of the article
}

treatment is applied [3]. Macroscopic vascular invasion into the main portal veins or their branches can be detected by CT or MRI, while microscopic vascular invasion is identifiable only by microscopy [4]. Therefore, early diagnosis PVTT of HCC is of paramount importance for the treatment of HCC patients.

Prothrombin induced by vitamin $\mathrm{K}$ absence-II (PIVK A-II) is a prothrombin (PT) precursor with no coagulation activity secreted from HCC cells, and it has been shown to be a predictor of microvascular invasion (MVI) [5, 6]. Serum levels of PIVKA-II have been demonstrated significantly increased in HCC patients and employed as a diagnostic marker in Asia $[6,7]$.

(C) The Author(s). 2019 Open Access This article is distributed under the terms of the Creative Commons Attribution 4.0 International License (http://creativecommons.org/licenses/by/4.0/), which permits unrestricted use, distribution, and 
Moreover, several studies have revealed that PIVKA-II serum levels were also significantly elevated in Eastern and European HCC patients $[6,8]$. According to some in vivo studies, PIVKA-II could induce the overexpression of vascular endothelial growth factor (VEGF) and it may promote vascular invasion $[9,10]$. Significantly higher PIVKA-II tissue expression was detected in HCC with microvascular invasion (MVI), as well as in serum [6]. Therefore, it could be employed as a predictor of microvascular invasion [6]. However, the role of PIVK A-II in the development of macrovascular invasion such as PVTT is not well demonstrated.

The present study aimed to detect the difference of PIVKA-II serum levels between HCC patients with PVTT and those without PVTT and evaluate the correlation between PIVKA-II and PVTT. In addition, we also investigated the correlation between serum levels of other HCC pathological characteristics such as AFP, D-Dimer and the development of PVTT.

\section{Patients and methods Patients}

Between March 2016 and October 2018, a total of 123 consecutive patients with HBV-related HCC with or without PVTT were enrolled in this study. HCC diagnose was based on typical radiologic results of HCC on two dynamic image examinations or one dynamic technique with serum $\alpha$-fetoprotein (AFP) level $\geq 200 \mathrm{ng} / \mathrm{ml}$ $[11,12]$. Clinicopathologcial and laboratory data were obtained from all subjects. Imaging examination such as contrast-enhanced computed tomography (CECT) and magnetic resonance imaging (CEMRI) accompanied by intraoperative or postoperative histopathology were employed to diagnose PVTT in HCC patients [13]. A filling defect in the portal vein or its branch on contrast-enhanced computed tomography or magnetic resonance imaging were used to distinguish PVTT or thrombus. There were 4 types of PVTT based on the PVTT range in the portal vein: Type I, tumor thrombus in the segmental branches of the portal vein or above; Type II, tumor thrombus extending to the right or the left portal vein; Type III, tumor thrombus extending to the main portal vein; and Type IV, tumor thrombus extending to the main portal vein and the superior mesenteric vein [14].

The study was approved by ethical committee of Provincial Hospital affiliated to Shandong University. Written informed consent was obtained from each subject.

\section{Serum sample collection and assays}

Peripheral blood samples were collected from each HCC patents right before surgeries or other treatments. The samples were spun and stored at $-80^{\circ} \mathrm{C}$ until laboratory tests.
Serum level of PIVKA-II was detected with a Lumipulse G PIVKA-II reagent kit (FUJIREBIO Inc., Japan) on a LUMI-PULSE g1200 automatic immune analyzer according to manufacturer's manual.

\section{Statistical analysis}

Continuous variables with normal distribution are presented as mean \pm standard deviation and were compared using Student's $t$ test. Categorical variables are expressed in absolute values and compared with the Chi-square test. Logistic Regression analysis was performed for independent variables of risk factors with PVTT in HCC patients, including gender, age, ALT, AST, PIVKA-II and D-dimer. Receiver operating characteristic (ROC) curve analysis was used to measure the diagnostic accuracy of PIVKA-II for the development of PVTT in HCC. All statistical analyses were accomplished using SPSS19.0 (IBM, Chicago, US). Statistical analysis was tested on two-sided settings and $P<0.05$ considered as statistically significant.

\section{Results}

Baseline characteristics and comparison of PIVKA-II levels in analysis group and validation group

Thirty-five HCC patients with PVTT and 88 HCC patients without PVTT were enrolled in the present study between March 2016 and October 2018. All the subjects were randomly divided into the analysis group $(N=73)$ and validation group $(N=50)$. The baseline characteristics of all subjects are summarized in Table 1 . The average age was 57.9 years, and $88.6 \%$ of the patients were male. Most HCC was accompanied with liver cirrhosis (85.4\%). All patients were BCLC stage A, B or C due to multiple nodules, PVTT or extrahepatic metastasis. Baseline characteristics were comparable between the two groups (Table 1).

\section{HCC patients with PVTT were detected with higher D- dimer level in analysis group}

Elevated D-dimer level was detected in HCC patients with PVTT compared with those of HCC patients without PVTT, as shown in Table 2. The mean level of D-dimer among HCC patients with PVTT was $2.12(0.27-15.61) \mathrm{mg} / \mathrm{ml}$, significantly higher than that of HCC patients without PVTT 0.56(0.10-13.95, $P=0.001)$ in analysis group (Fig. 1). Moreover, univariate analysis showed that high serum $\mathrm{D}$-dimer level $(\mathrm{OR}=1.22$, 95\% CI 1.02-1.45) was an independent risk factor for development of PVTT.

\section{Diagnostic value of D-DIMER for PVTT in HCC patients of analysis group and validation group}

A ROC curve was conducted to assess the diagnostic value of D-dimer for PVTT development in HCC patients, HCC patients without PVTT were enrolled as 
Table 1 Baseline characteristics of the study population

\begin{tabular}{|c|c|c|c|c|}
\hline Variables & All patients & Analysis group & Validation group & $P$ value \\
\hline Sex F/M & $14 / 109$ & $9 / 64$ & $5 / 45$ & 0.69 \\
\hline Age (years) & $57.94 \pm 10.96$ & $57.88 \pm 10.03$ & $58.02 \pm 12.29$ & 0.94 \\
\hline $\operatorname{ALT}(U / L)$ & $30(8-471.1)$ & $27(10-261)$ & $35.5(8-471.1)$ & 0.30 \\
\hline AST (U/L) & $47(13-544)$ & $38(13-291)$ & $52.5(16-544)$ & 0.06 \\
\hline TBiL ( $\mu \mathrm{mol} / \mathrm{L})$ & $18.3(8.5-457)$ & $17.8(8.5-457.0)$ & $20.0(8.9-113.2)$ & 0.41 \\
\hline ALB (g/L) & $36.53 \pm 4.91$ & $36.98 \pm 4.78$ & $35.87 \pm 5.06$ & 0.22 \\
\hline D-Dimer (mg/L) & $0.80(0.10-15.61)$ & $1.1(0.27-15.61)$ & $0.72(0.10-13.95)$ & 0.23 \\
\hline PTA (\%) & $80.62 \pm 14.57$ & $82.31 \pm 12.92$ & $78.14 \pm 16.51$ & 0.12 \\
\hline $\operatorname{PLT}\left(\times 10^{9} / \mathrm{L}\right)$ & $162.09 \pm 99.10$ & $161.96 \pm 102.87$ & $162.28 \pm 94.37$ & 0.97 \\
\hline AFP & $441.06(1.33-1210.0)$ & 393.09 (1.33-1210.0) & $511.09(1.5-1210)$ & 0.38 \\
\hline PIVKA-II (mAU/ml) & $278.13(1.6-75,000.00)$ & $196.12(12-75,000.00)$ & $514.86(1.6-75,000)$ & 0.45 \\
\hline \multicolumn{5}{|l|}{ Liver cirrhosis } \\
\hline YES/NO & $105 / 18$ & $30 / 5$ & $75 / 13$ & 0.95 \\
\hline \multicolumn{5}{|l|}{ Child-Pugh grade } \\
\hline A & 68 & 37 & 31 & \multirow[t]{2}{*}{0.27} \\
\hline B & 55 & 36 & 19 & \\
\hline \multicolumn{5}{|l|}{ PVTT, type } \\
\hline 1 & 34 & 21 & 13 & \multirow[t]{4}{*}{0.91} \\
\hline$\|$ & 51 & 30 & 21 & \\
\hline$\|$ & 19 & 12 & 7 & \\
\hline IV & 19 & 10 & 9 & \\
\hline \multicolumn{5}{|l|}{ BCLC stage } \\
\hline A & 12 & 9 & 3 & \multirow[t]{3}{*}{0.48} \\
\hline B & 76 & 43 & 33 & \\
\hline C & 35 & 21 & 14 & \\
\hline $\mathrm{HBsAg}(\mathrm{COI})$ & $4578(12.2-8657.0)$ & $4512(12.2-7542.0)$ & 5221.5 (35-8657.0) & 0.38 \\
\hline \multicolumn{5}{|l|}{ HBeAg } \\
\hline Positive & 55 & 32 & 23 & \multirow[t]{2}{*}{0.81} \\
\hline Negative & 68 & 41 & 27 & \\
\hline HBV-DNA (IU/L) & $7500.0\left(0.0-4.16 \times 10^{7}\right)$ & $5800.0\left(0.0-4.16 \times 10^{7}\right)$ & $21,750.0\left(0.0-3.26 \times 10^{7}\right)$ & 0.46 \\
\hline
\end{tabular}

control in analysis group. To identify cutoff values that could best distinguish PVTT in HCC patients of analysis group, ROC curves were employed. The AUROC of D-dimer was 0.75 (95\%CI $0.63-0.87, P=0.001)$, However, AUROC of D-dimer in validation group was 0.57 (95\%CI 0.39-0.75, $P=0.43$ ).

\section{Elevated PIVKA-II level was detected in HCC patients with PVTT in analysis group}

PIVKA-II level as well as other laboratory data of HCC patients with PVTT in analysis group were compared with those of HCC patients without PVTT, as shown in Table 2. The median level of PIVKA-II among HCC patients with PVTT was 995.80(12-75,000.00) $\mathrm{mAU} / \mathrm{ml}$, significantly higher than that of $\mathrm{HCC}$ patients without
PVTT 94.87(13-75,000) $\mathrm{mAU} / \mathrm{ml}$ with $P=0.003$ (Fig. 2). Univariate analysis showed that high PIVKA-II level $(\mathrm{OR}=1.95$, 95\%CI 1.23-3.09) was an independent risk factor for development of PVTT.

\section{Diagnostic value of PIVKA-II for PVTT in HCC patients of analysis group}

A ROC curve was conducted to assess the diagnostic value of PIVKA-II for PVTT development in HCC patients, HCC patients without PVTT were enrolled as control for analysis. To identify cutoff values that could best distinguish PVTT in HCC patients from controls, ROC curves were plotted. The AUROC of PIVKA-II was 0.73 (95\%CI $0.59-0.86, P=0.003)$. The optimal cutoff 
Table 2 Baseline characteristics of analysis group

\begin{tabular}{|c|c|c|c|c|}
\hline Variables & Analysis group & HCC patients with PVTT & Controls & $P$ value \\
\hline Sex F/M & $9 / 64$ & $4 / 17$ & $5 / 47$ & 0.27 \\
\hline Age (years) & $57.88 \pm 10.03$ & $55.33 \pm 9.62$ & $58.90 \pm 10.10$ & 0.17 \\
\hline ALT & $27(10-261.1)$ & $39(10-147)$ & $24.5(10-261.1)$ & 0.97 \\
\hline AST & $38(13-291)$ & $47(13-105)$ & $36.5(17-291)$ & 0.38 \\
\hline TBiL & $17.8(8.5-457)$ & $22.4(9.4-407.9)$ & $17.35(8.5-457.0)$ & 0.26 \\
\hline ALB & $36.98 \pm 4.78$ & $35.96 \pm 4.27$ & $37.39 \pm 4.96$ & 0.25 \\
\hline D-Dimer (mg/L) & $1.1(0.27-15.61)$ & $2.12(0.27-15.61)$ & $0.56(0.10-13.95)$ & 0.001 \\
\hline PTA & $82.31 \pm 12.92$ & $79.84 \pm 11.34$ & $83.31 \pm 13.48$ & 0.30 \\
\hline $\operatorname{PLT}\left(\times 10^{9} / \mathrm{L}\right)$ & $161.96 \pm 102.87$ & $173.57 \pm 118.47$ & $157.27 \pm 96.73$ & 0.54 \\
\hline AFP & 393.09 (1.33-1210.0) & 469.17(3.10-1210) & $362.36(1.7-1210)$ & 0.21 \\
\hline PIVKA-II (mAU/ml) & $196.12(12-75,000.00)$ & $995.8(12-75,000.00)$ & $94.87(13-70,997.0)$ & 0.003 \\
\hline \multicolumn{5}{|l|}{ Child-Pugh class } \\
\hline A & 37 & 9 & 28 & \multirow[t]{2}{*}{0.45} \\
\hline B & 36 & 12 & 24 & \\
\hline $\mathrm{HBsAg}(\mathrm{COI})$ & $4512(12.2-7542.0)$ & $5067.0(62.33-7070.0)$ & 4106 (12.2-7542.0) & 0.35 \\
\hline \multicolumn{5}{|l|}{ HBeAg } \\
\hline Positive & 32 & 11 & 21 & \multirow[t]{2}{*}{0.43} \\
\hline Negative & 41 & 10 & 31 & \\
\hline HBV-DNA & $5800.0\left(0.0-4.16 \times 10^{7}\right)$ & $4800.0\left(0.0-2.99 \times 10^{6}\right)$ & $6285.0\left(0.0-4.16 \times 10^{7}\right)$ & 0.81 \\
\hline
\end{tabular}

value of PIVKA-II was $221.26 \mathrm{mAU} / \mathrm{ml}$, with a sensitivity of $83.70 \%$ and a specificity of $69.20 \%$ (Fig. 3).

Validation of PIVKA-II for PVTT detection in HCC patients To validate the diagnostic value of PIVKA-II in development of PVTT in HCC patients, a ROC curve was conducted in validation group. ROC curves were plotted. The AUROC of PIVKA-II was 0.84 (95\%CI 0.70-0.97, P $<0.01)$. The sensitivity and specificity of cutoff value $221.26 \mathrm{mAU} / \mathrm{ml}$ were 85.71 and $55.56 \%$, respectively.

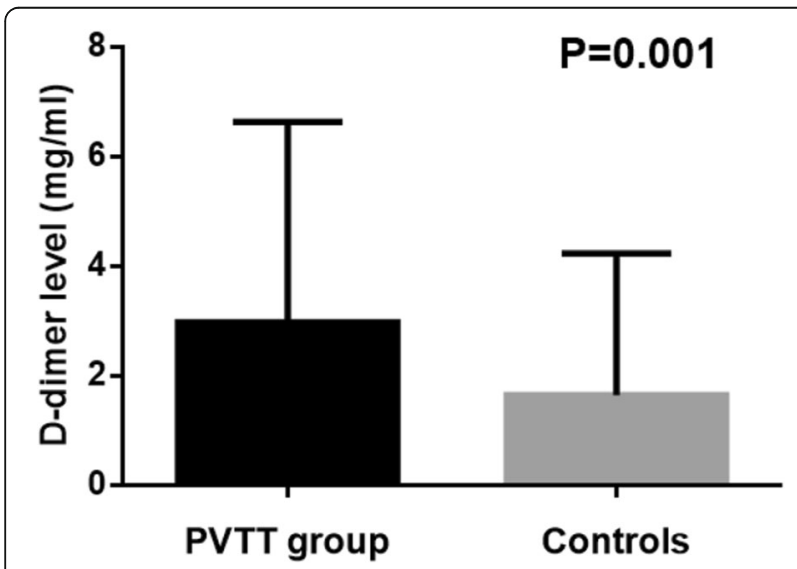

Fig. 1 Difference of D-dimer plasma levels between HCC patients with PVTT and controls in analysis group. PVTT group:group of HCC patients with PVTT. Controls: group of HCC patients without PVTT

\section{Discussion}

PVTT is an important predictor and prognostic factor for recurrence of HCC patients, which is occurred in about $10-40 \%$ patients with $\mathrm{HCC}$ at the time of diagnosis [15-17]. PVTT could increase portal venous pressure, decrease the blood flow to the liver, which can result in gastrointestinal hemorrhage or liver failure. Therefore, PVTT is a major factor to influence the median OS duration of postoperative $\mathrm{HCC}$ patients [15]. In the present study, we found that serum level of PIVKA-II was associated with the development of PVTT in HCC patients.

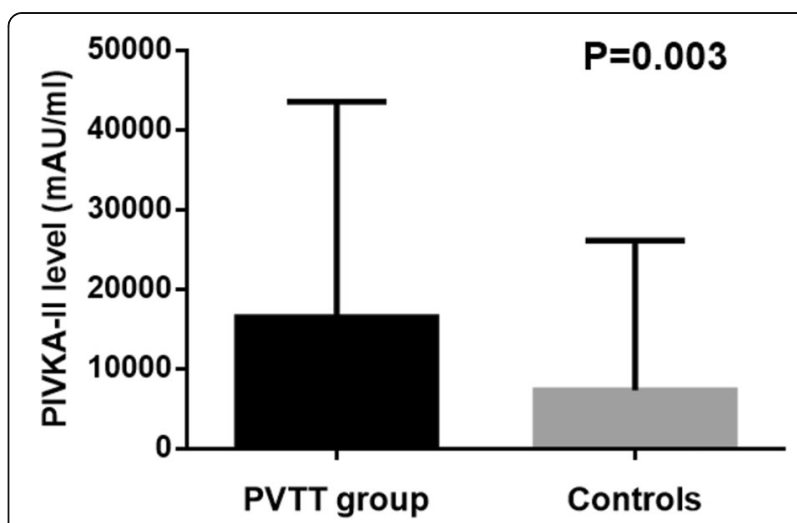

Fig. 2 Difference of PIVKA-II plasma levels between HCC patients with PVTT and controls in analysis group. PVTT group:group of HCC patients with PVTT. Controls: group of HCC patients without PVTT 


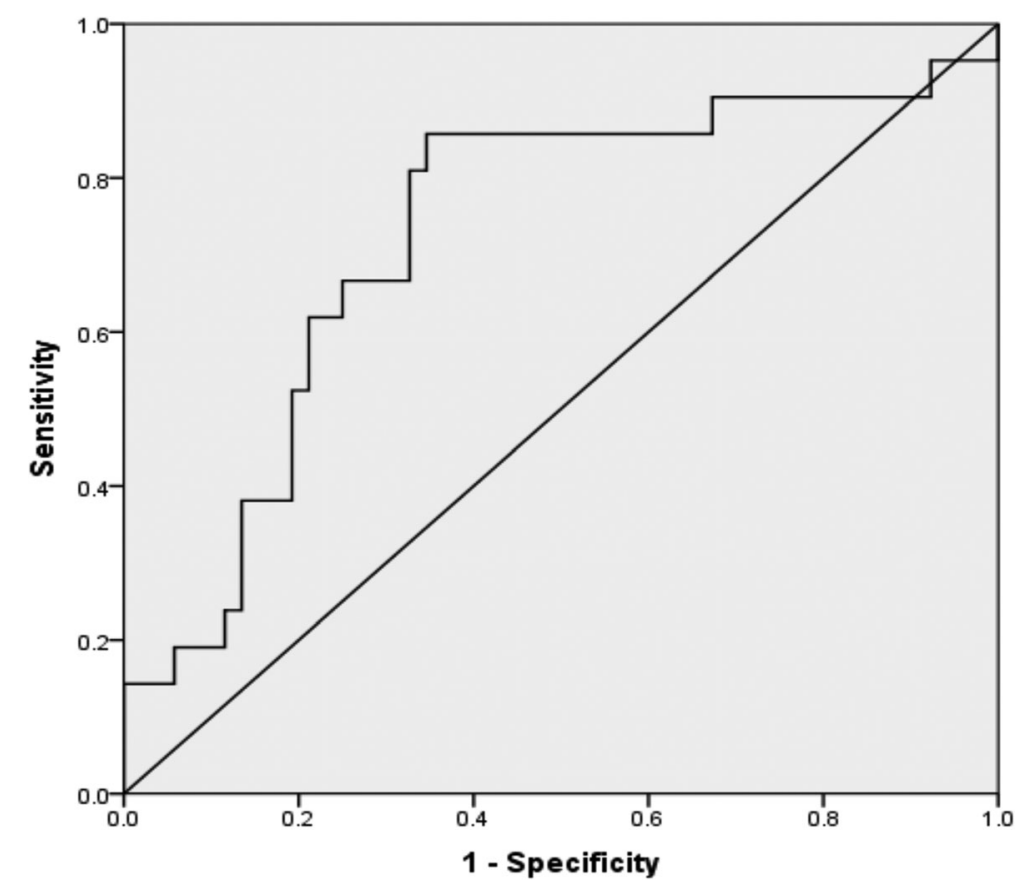

Fig. 3 Diagnostic values of PIVKA-II in HCC patients with PVTT. The AUROC of PIVKA- II to diagnose HCC patients with PVTT was 0.70 (95\% $\mathrm{Cl}$ 0.60-0.81). For the diagnosis of PVTT in HCC, PIVKA-II had a sensitivity of $77.1 \%$ and a specificity of $63.6 \%$ at a cutoff of 327.69 $\mathrm{mAU} / \mathrm{ml}$.

Up to date, PIVKA-II has been employed as an alternative tumor marker of AFP for HCC diagnose including early HCC, with a cut-off value of $40 \mathrm{mAU} / \mathrm{ml}[7,18$, 19]. The current study showed that PIVKA-II could also be a marker of PVTT in HCC patients. For that, HCC patients with PVTT demonstrate significantly higher PIVKA-II level compared with HCC patients without PVTT and the optimal cutoff value of PIVK A-II in diagnostic of PVTT in HCC patients was $221.26 \mathrm{mAU} / \mathrm{ml}$, with a sensitivity of $83.70 \%$ and a specificity of $69.20 \%$. In line with our findings, other researchers also found that serum PIVKA-II levels in HCC patients elevated in parallel with the progression of HCC, which are associated with poor prognosis [20]. Moreover, serum PIVKA-II levels in HCC patients significantly elevated according to BCLC stage, while AFP levels did not [21].

The present study indicated that serum PIVKA-II level could be employed as a biomarker for PVTT development HCC patients. In combine with other researches, we hypothesize that PIVKA-II may play a role in the development of PVTT in HCC patients along with the prognosis. According to a previous research, PIVKA-II could promote proliferation and migration of vascular endothelial cells, elevated serum level of PIVKA-II is associated with microvascular invasion [5]. For instance, Kim et al., reported that elevated serum PIVKA-II level was associated with microvascular invasion in small HCC $(<2 \mathrm{~cm})$, and serum PIVKA-II level $\geq 200 \mathrm{mAU} /$ $\mathrm{mL}$ was a predisposing factor for tumor recurrence after surgery [22]. Another recent meta-analysis study revealed that higher PIVKA-II could be a predictor of unfavorable overall survival in hepatocellular carcinoma patients receiving curative ablation [23]. Moreover, PIVKA-II has been employed as an important factor in consideration of liver transplantation for HCC patients [24].

Another interesting result in the current study indicated that D-Dimer was an independent risk factor for development of PVTT. Not only in PVTT patients, several studies revealed that high D-Dimer levels were also found in both liver cirrhosis and HCC patients [25]. In consistent with our findings, Liu, et al., also reported that D-dimer level elevated in advanced HCC patients and predicted poor prognosis $[25,26]$. We hypothesize that thrombotic invasion of portal or hepatic vein may be involved in development of PVTT and result in high D-dimer level. D-dimer may serve as a complementary indicator that could detect the development of PVTT in HCC patients.

To our best knowledge, the present study shows for the first time that PIVKA is useful for predicting the risk of PVTT. Further studies are necessary to validate this correlation. 


\section{Acknowledgements}

Declared none.

\section{Funding}

Medical and health science and technology development plan of Shandong Province (Grant No.2016WS0420)

\section{Availability of data and materials}

Authors can confirm all relevant data are included in the article and materials are available on request from the authors.

\section{Authors' contributions}

$J H$ and YC designed the study, ST and JZ edited the final version of the manuscript; $T L, Y Y$ and $J L$ conducted the sample analysis and drafted the paper; XT and LW contributed to the statistical analyses; TL, MK and SG provided the clinical samples. All authors read and approved the final manuscript.

\section{Ethics approval and consent to participate}

This study was approved the institutional ethics committee of Provincial Hospital affiliated to Shandong University. Written informed consent was obtained from each subject.

\section{Consent for publication}

Not applicable.

\section{Competing interests}

The authors declare that they have no competing interests.

\section{Publisher's Note}

Springer Nature remains neutral with regard to jurisdictional claims in published maps and institutional affiliations.

\section{Author details}

'Department of Gastroenterology, Provincial Hospital Affiliated to Shandong University, 324, Jing 5 Rd, Jinan 250021, Shandong Province, China. ${ }^{2}$ Department of Gastrointestinal surgery, Provincial Hospital affiliated to Shandong University, Jing 5 Road, Jinan, People's Republic of China ${ }^{3}$ Department of rehabilitation, Provincial Hospital affiliated to Shandong University, Jing 5 Road, Jinan, People's Republic of China. ${ }^{4}$ Department of Gastroenterology, Shouguang peoples' Hospital, No.45, health street, Shouguang city, Weifang city, People's Republic of China.

Received: 6 January 2019 Accepted: 17 April 2019

Published online: 14 May 2019

\section{References}

1. Tsuchiya N, Sawada Y, Endo I, Saito K, Uemura Y, Nakatsura T. Biomarkers for the early diagnosis of hepatocellular carcinoma. World J Gastroenterol. 2015; 21:10573-83.

2. Park JW, Chen M, Colombo M, Roberts LR, Schwartz M, Chen PJ, et al. Global patterns of hepatocellular carcinoma management from diagnosis to death: the BRIDGE study. Liver Int. 2015;35:2155-66

3. Villa E, Moles A, Ferretti I, Buttafoco P, Grottola A, Del Buono M, et al. Natural history of inoperable hepatocellular carcinoma: estrogen receptors' status in the tumor is the strongest prognostic factor for survival. Hepatology. 2000;32:233-8.

4. Roayaie S, Blume IN, Thung SN, Guido M, Fiel Ml, Hiotis S, et al. A system of classifying microvascular invasion to predict outcome after resection in patients with hepatocellular carcinoma. Gastroenterology. 2009;137:850-5.

5. Fujikawa T, Shiraha H, Ueda N, Takaoka N, Nakanishi Y, Matsuo N, et al. Desgamma-carboxyl prothrombin-promoted vascular endothelial cell proliferation and migration. J Biol Chem. 2007;282:8741-8.

6. Poté N, Cauchy F, Albuquerque M, Voitot H, Belghiti J, Castera L, et al. Performance of PIVKA-II for early hepatocellular carcinoma diagnosis and prediction of microvascular invasion. J Hepatol. 2015;62:848-54.

7. Wang X, Zhang W, Liu Y, Gong W, Sun P, Kong X, et al. Diagnostic value of prothrombin induced by the absence of vitamin $\mathrm{K}$ or antagonist-II (PIVKA-II) for early stage HBV related hepatocellular carcinoma. Infect Agent Cancer. 2017;12:47.
8. Abd El Gawad IA, Mossallam Gl, Radwan NH, Elzawahry HM, Elhifnawy NM. Comparing prothrombin induced by vitamin K absence-II (PIVKA-II) with the oncofetal proteins glypican-3, alpha feto protein and carcinoembryonic antigen in diagnosing hepatocellular carcinoma among Egyptian patients. J Egypt Natl Canc Inst. 2014;26:79-85.

9. Wang SB, Cheng YN, Cui SX, Zhong JL, Ward SG, Sun LR, et al. Des-gammacarboxy prothrombin stimulates human vascular endothelial cell growth and migration. Clin Exp Metastasis. 2009;26:469-77.

10. Gao FJ, Cui SX, Chen MH, Cheng YN, Sun LR, Ward SG, et al. Des-gammacarboxy prothrombin increases the expression of angiogenic factors in human hepatocellular carcinoma cells. Life Sci. 2008:83:815-20.

11. Song DS, Bae SH. Changes of guidelines diagnosing hepatocellular carcinoma during the last ten-year period. Clin Mol Hepatol. 2012;18:258-67.

12. Ministry of Health of the People's Republic of China. Diagnosis, management, and treatment of hepatocellular carcinoma. J Clin Hepat (Chinese). 2011;27:1141-3.

13. Zhang ZM, Lai EC, Zhang C, Yu HW, Liu Z, Wan BJ, et al. The strategies for treating primary hepatocellular carcinoma with portal vein tumor thrombus. Int J Surg. 2015;20:8-16.

14. Peng SY, Wang XA, Huang CY, Li JT, Hong DF, Wang YF, et al. Better surgical treatment method for hepatocellular carcinoma with portal vein tumor thrombus. World J Gastroenterol. 2018;24:4527-35.

15. Quirk M, Kim Y, Saab S, Wolfgang LE. Management of hepatocellular carcinoma with portal vein thrombosis. World J Gastroenterol. 2015;21:3462-71.

16. Minagawa M, Makuuchi M. Treatment of hepatocellular carcinoma accompanied by portal vein tumor thrombus. World J Gastroenterol. 2006;12:7561-7.

17. Chan SL, Mo FK, Johnson PJ, Liem GS, Chan TC, Poon MC, et al. Prospective validation of the Chinese University prognostic index and comparison with other staging systems for hepatocellular carcinoma in an Asian population. J Gastroenterol Hepatol. 2011;26:340-7.

18. Li C, Zhang Z, Zhang P, Liu J. Diagnostic accuracy of des-gamma-carboxy prothrombin versus alpha-fetoprotein for hepatocellular carcinoma: a systematic review. Hepatol Res. 2014;44:E11-5.

19. Yu R, Tan Z, Xiang X, Dan Y, Deng G. Effectiveness of PIVKA-II in the detection of hepatocellular carcinoma based on real-world clinical data. BMC Cancer. 2017;17:608

20. Kang SH, Kim DY, Jeon SM, Ahn SH, Park JY, Kim SU, et al. Clinical characteristics and prognosis of hepatocellular carcinoma with different sets of serum AFP and PIVKA-II levels. Eur J Gastroenterol Hepatol. 2012;24:849-56.

21. Ma XL, Zhu J, Wu J, Tian L, Gao YY, Zhang CY, et al. Significance of PIVKA-II levels for predicting microvascular invasion and tumor cell proliferation in Chinese patients with hepatitis B virus-associated hepatocellular carcinoma. Oncol Lett. 2018;15:8396-404.

22. Kim JM, Hyuck C, Kwon D, Joh JW, Lee JH, Paik SW, et al. Protein induced by vitamin $\mathrm{K}$ antagonist-II (PIVKA-II) is a reliable prognostic factor in small hepatocellular carcinoma. World J Surg. 2013:37:1371-8.

23. Zhang D, Liu Z, Yin X, Qi X, Lu B, Liu Y, et al. Prognostic value of PIVKA-II in hepatocellular carcinoma patients receiving curative ablation: a systematic review and meta-analysis. Int J Biol Markers. 2018;33:266-74.

24. Lee KW, Suh SW, Choi Y, Jeong J, Yi NJ, Kim H, et al. Macrovascular invasion is not an absolute contraindication for living donor liver transplantation. Liver Transpl. 2017;23:19-27.

25. Spadaro A, Tortorella V, Morace C, Fortiguerra A, Composto P, Bonfiglio C, et al. High circulating D-dimers are associated with ascites and hepatocellular carcinoma in liver cirrhosis. World J Gastroenterol. 2008;14:1549-52.

26. Liu Z, Guo H, Gao F, Shan Q, Li J, Xie H, et al. Fibrinogen and D-dimer levels elevate in advanced hepatocellular carcinoma: high pretreatment fibrinogen levels predict poor outcomes. Hepatol Res. 2017;47:1108-17. 